\title{
EFEITOS DA NEBULOSIDADE NA ABSORÇÃO DE CARBONO EM UMA CULTURA DE MILHO EM CRUZ ALTA, RS
}

Leonardo J. G. Aguiar ${ }^{1}$, José M. N. Costa ${ }^{1}$, Débora R. Roberti ${ }^{2}$, Williams P. M. Ferreira ${ }^{3}$, Claudio Teichrieb ${ }^{2}$, Graciela R. Fischer ${ }^{4}$, Evandro C. Oliveira ${ }^{5}$, Osvaldo L. L. Morais ${ }^{2}$

\author{
${ }^{1}$ Embrapa Amapá, AP, Brasil \\ ${ }^{2}$ Universidade Federal de Santa Maria, RS, Brasil \\ ${ }^{3}$ Embrapa Milho e Sorgo, MG, Brasil \\ ${ }^{4}$ Universidade Federal de Pelotas, RS, Brasil \\ ${ }^{5}$ Instituto Federal do Espírito Santo, ES, Brasil \\ veraneiro@yahoo.com.bbr
}

\section{RESUMO}

Foram realizadas medições no período de 11 de novembro de 2010 a 19 de fevereiro de 2011 de variáveis meteorológicas e fluxo de dióxido de carbono $\left(\mathrm{CO}_{2}\right)$, através da técnica de covariância dos vórtices turbulentos, com o intuito de analisar a influência da nebulosidade na absorção do $\mathrm{C}$ em uma cultura de milho em Cruz Alta, RS. Observou-se maior absorção de $\mathrm{C}$ a níveis intermediários de cobertura de nuvens.

\section{SUMMARY}

Measurements were performed from 11 November 2010 to 19 February 2011 of meteorological variables and carbon dioxide $\left(\mathrm{CO}_{2}\right)$ flux using the technique of eddy covariance in order to analyze the influence of cloudiness on the absorption of $\mathrm{C}$ in a maize crop in Cruz Alta, RS. We observed a higher absorption of $\mathrm{C}$ at intermediate levels of cloud cover.

\section{INTRODUÇÃO}

A fotossíntese é fortemente relacionada à disponibilidade de radiação fotossinteticamente ativa (PAR). Recentes estudos teóricos e observacionais têm demonstrado que a fotossíntese é mais eficiente sob condições de luz difusa (OLIVEIRA et al., 2007), com o céu parcialmente nublado.

Entender como a nebulosidade afeta a fotossíntese e, consequentemente, a absorção de carbono em uma cultura de milho no Rio Grande do Sul é de grande importância para o desenvolvimento/adaptação de modelos de ecossistemas agrícolas para o Sul do Brasil.

Objetivou-se nesse estudo analisar a influência da nebulosidade na absorção de C em uma cultura de milho.

\section{MATERIAL E MÉTODOS}

O presente trabalho foi realizado no sítio experimental pertencente à Rede SULFLUX, situado na FUNDACEP (283' S; 5340' O; 409 m de altitude), localizada no município de Cruz Alta, no Estado do Rio Grande do Sul.

Foram realizadas medições contínuas no período de 11 de novembro de 2010 a 19 de fevereiro de 2011 de irradiância solar global (Rs, piranômetro LI-COR (LI200SA)) e PAR 
(PAR, quantum LI-COR (LI190SA)) acima do dossel da cultura, bem como umidade volumétrica do solo ( $\mathrm{U}_{\text {solo }}$, Sonda helicoidal TDR (Time Domain Reflectometry)) na camada de 0 a $30 \mathrm{~cm}$ de profundidade.

As medições de fluxo de $\mathrm{CO}_{2}$ foram realizadas através da técnica de covariância de vórtices turbulentos, utilizando um analisador de gás por infravermelho de caminho aberto (LI-7500), fabricado pela LI-COR Inc., e um anemômetro sônico (CSAT3 3-D), fabricado pela Campbell Scientific Instrument, ambos instalados a 2,5 metros de altura da superfície.

Para medir a área foliar, foi utilizado o integrador de área foliar LI3000A, fabricado pela LI-COR Inc, com periodicidade média de 15 dias.

\section{RESULTADOS E DISCUSSÃO}

Para avaliar os efeitos da nebulosidade na absorção de $\mathrm{C}$ pela cultura do milho, foram utilizados nove dias de dados após o fechamento do dossel (Tabela 1), os quais foram separados em três classes de cobertura de nuvens: primeira $(\mathrm{C} 1), \mathrm{k}_{\mathrm{t}}$ (razão entre Rs e a radiação solar extraterrestre) variando entre 0,5 e 0,6 ; segunda $(C 2), \mathrm{k}_{\mathrm{t}}$ variando entre 0,6 e 0,7 ; e terceira $(\mathrm{C} 3), \mathrm{k}_{\mathrm{t}}$ variando entre 0,7 e 0,8 . Os dias utilizados foram escolhidos devido à proximidade nas condições de umidade do solo. A C1 apresentou uma diminuição de aproximadamente $13 \%$ na absorção de $\mathrm{C}$ em relação a $\mathrm{C} 3$, acompanhando a redução de cerca de $27 \%$ da PAR.

Tabela 1. Valores diários do índice de claridade $\left(\mathrm{k}_{\mathrm{t}}\right.$, adimensional), fluxo de carbono $\left(\mathrm{F}_{\mathrm{c}}, \mathrm{gC}\right.$ $\mathrm{m}^{-2} \mathrm{~d}^{-1}$ ), índice de área foliar (IAF, $\mathrm{m}^{2} \mathrm{~m}^{-2}$ ), radiação fotossinteticamente ativa (PAR, MJ $\mathrm{m}^{-2}$ $\left.\mathrm{d}^{-1}\right)$ e umidade do solo $\left(\mathrm{U}_{\text {solo }}, \mathrm{m}^{3} \mathrm{~m}^{-3}\right)$

\begin{tabular}{lllllllc}
\hline Clas-se & Dia Juliano & DAE & kt & FC & IAF & PAR & Usolo \\
& & & & & & & \\
\hline \multirow{2}{*}{ C1 } & 355 & 73 & 0,56 & $-8,93$ & 4,17 & 11,12 & 0,45 \\
& 3 & 86 & 0,55 & $-7,35$ & 4,39 & 10,29 & 0,39 \\
& 4 & 87 & 0,53 & $-7,00$ & 4,40 & 10,33 & 0,41 \\
$\mathbf{3} 2$ & 356 & 74 & 0,62 & $-10,79$ & 4,20 & 12,07 & 0,43 \\
& 364 & 82 & 0,69 & $-10,27$ & 4,36 & 13,46 & 0,33 \\
& 365 & 83 & 0,66 & $-10,03$ & 4,37 & 12,81 & 0,32 \\
$\mathbf{3} 3$ & 343 & 61 & 0,76 & $-8,34$ & 3,37 & 14,34 & - \\
& 349 & 67 & 0,76 & $-8,92$ & 3,88 & 14,48 & 0,44 \\
& 360 & 78 & 0,77 & $-9,53$ & 4,30 & 14,62 & 0,38 \\
\hline
\end{tabular}

$\mathrm{Na} \mathrm{C} 2$, apesar das menores quantidades de umidade do solo $(12,2 \%)$ e da PAR $(\approx 12 \%)$, devido à maior cobertura de nuvens, a absorção de carbono foi cerca de $16 \%$ superior em relação a $\mathrm{C} 3$, o que provavelmente ocorreu devido a maior absorção de $\mathrm{CO}_{2}$ encontrada em dias mais nublados, menor $\mathrm{k}_{\mathrm{t}}$, para uma mesma quantidade de PAR (Figura $1)$.

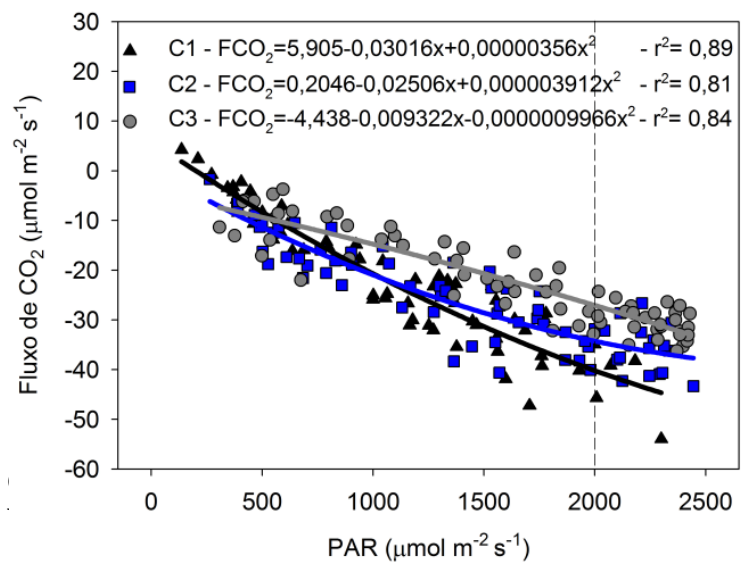

Figura 1. Relação entre a PAR e o fluxo de $\mathrm{CO}_{2}$ nas três classes avaliadas. 
Essa maior absorção do $\mathrm{CO}_{2}$ em dias mais nublados ocorre provavelmente devido à maior quantidade de radiação que chega a níveis mais baixos do dossel, em que as folhas são mais eficientes no uso da radiação (URBAN et al., 2007). Gu et al. (1999) relatam que para que a mesma quantidade de PAR obtida em dias claros ocorra em dias nublados geralmente o sol deve estar em elevações maiores, o que favorece a penetração da radiação no dossel.

\section{CONCLUSÕES}

A magnitude dos fluxos de $\mathrm{C}$ da cultura apresentou dependência com relação à nebulosidade, havendo maior absorção de carbono a níveis intermediários de cobertura de nuvens, ocasionada pela maior quantidade de radiação que chega a níveis mais baixos no dossel.

\section{AGRADECIMENTOS}

Agradecemos aos estudantes bolsistas da Universidade Federal de Santa Maria pela coleta dos dados. O primeiro autor agradece a CAPES pelo apoio financeiro dado através da concessão de bolsa de doutorado.

\section{BIBLIOGRAFIA CITADA}

GU, L.; et al. Responses of net ecosystem exchanges of carbon dioxide to changes in cloudiness: Results from two North American deciduous forests, Journal of Geophysical Research, 104, 31,421-31,434, 1999.

OLIVEIRA, P. H. F.; et al. The effects of biomass burning aerosols and clouds on the $\mathrm{CO}_{2}$ flux in Amazonia. Tellus B., v. 59, p. 338-349, 2007.

URBAN, O.; et al. Ecophysiological controls over the net ecosystem exchange of mountain spruce stand. Comparison of the response in direct vs. diffuse solar radiation. Global Change Biology, v. 13, p. 157-168, 2007. 